\title{
Decoherence of intermolecular entanglement in exchange-coupled nanomagnets
}

\author{
A. Szallas ${ }^{1,2}$ and F. Troiani ${ }^{1}$ \\ ${ }^{1}$ S3, Istituto Nanoscienze-CNR, Via G. Campi 213A, I-41125 Modena \\ ${ }^{2}$ Dipartimento di Fisica, Università di Modena e Reggio Emilia, via G. Campi 213/a, 41100 Modena, Italy
}

(Dated: August 9, 2021)

\begin{abstract}
We theoretically investigate the hyperfine-induced decoherence in a pair of spin-cluster qubits, consisting of two exchange-coupled heterometallic wheels. We identify two distinct regimes in the decoherence of intermolecular entanglement and show that this can be substantially recovered through dynamical decoupling. Different chemical elements and physical processes are responsible for the decoherence of the singlet-triplet superposition, resulting in a wider tunability of its decoherence time.
\end{abstract}

PACS numbers: 75.50.Xx, 03.65.Yz, 03.67.Bg

Molecular nanomagnets (MNs) represent a rich class of spin clusters, whose properties can be widely tailored by chemical synthesis [2]. Weak interactions between well defined molecular units have also been demonstrated [3] and can be made tunable by introducing different intermolecular linkers 4. This has recently allowed to demonstrate equilibrium-state entanglement between pairs of heterometallic wheels [1. Quantum mechanical features, such as free [5, 6] and forced [7 9] oscillations, have also emerged in the coherent dynamics of MNs. The tunable coupling between nanomagnets, combined with the coherent manipulation of their spin state by pulsed EPR,

(a)
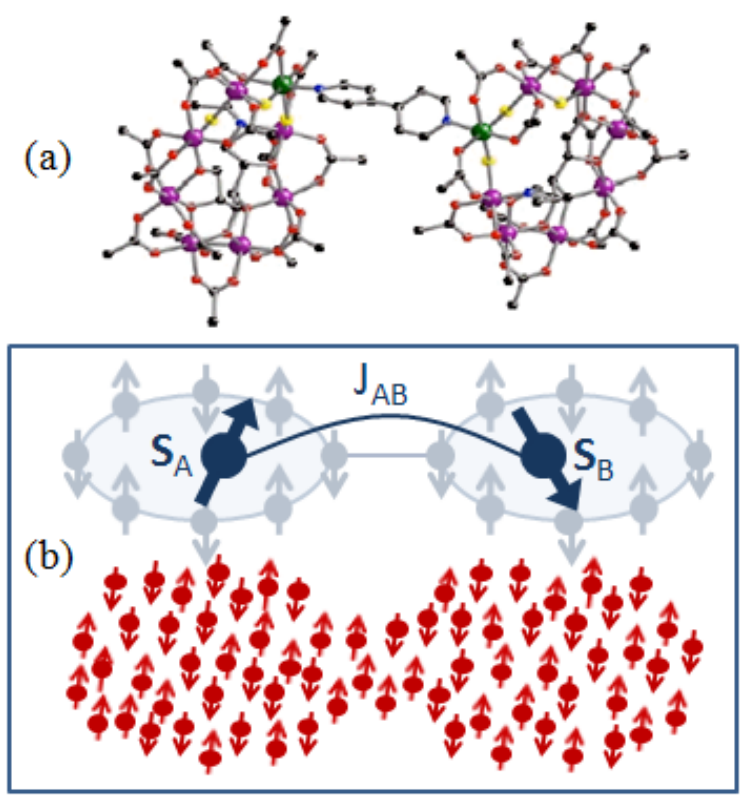

FIG. 1: (color online) (a) Chemical structure of the $\left(\mathrm{Cr}_{7} \mathrm{Ni}\right)_{2}$ dimer 1], as determined by X-ray diffraction: $\mathrm{Cr}$ (purple), $\mathrm{Ni}$ (green), $\mathrm{F}$ (yellow), $\mathrm{O}$ (orange), $\mathrm{C}$ (black), $\mathrm{N}$ (cyan); the $\mathrm{H}$ nuclei have been omitted for the sake of clarity. (b) Schematics of the spin system and environment. The state of the two electron-spin rings (faint blue), is mapped onto that of two exchange-coupled $1 / 2$ spins, $S_{A}$ and $S_{B}$ (blue). The electron spins interact with a bath of $N_{n}=312$ nuclear spins (red). will possibly enable the experimental investigation and the controlled generation of intermolecular coherence and entanglement. Besides being of fundamental interest, these issues are pivotal to the implementation of quantum information processing with MNs [10 13].

The main sources of electron spin decoherence in MNs are represented by intermolecular dipole-dipole interactions [14, spin-phonon [15] and hyperfine coupling [16, 17. While the former two contributions can be limited by dilution of the molecular ensemble and low temperatures [5], the suppression of hyperfine-induced decoherence might require more sophisticated dynamical decoupling techniques [18. In spite of its fundamental and applicative interest, still little is known on intermolecular coherence in coupled nanomagnets and on its robustness with respect to hyperfine interactions. Here we address this problem in the case of two spincluster qubits, each consisting of a $\mathrm{Cr}_{7} \mathrm{Ni}$ heterometallic wheel (Fig. 1(a)) 19. Being only the ground-state doublet of each wheel involved in the low-temperature dynamics, the electron spin state of the $\left(\mathrm{Cr}_{7} \mathrm{Ni}\right)_{2}$ dimer can be mapped onto that of two exchange-coupled spins $S_{A}=S_{B}=1 / 2$ (Fig. 1(b)). Essentially, decoherence is due to the dependence of the slow nuclear bath dynamics on the state of the electron spins, and to the resulting correlations between electron and nuclear spins. The features that characterize the decoherence of different linear superpositions can however remarkably differ from one another [20]. We indeed find similar differences between the decoherence of two prototypical linear superpositions $\left(\left|\Phi_{1}\right\rangle+\left|\Phi_{2}\right\rangle\right) / \sqrt{2}$, with $\left|\Phi_{i}\right\rangle$ the electron-spin eigenstates of the dimer. The first linear superposition, with components $\left|\Phi_{1}\right\rangle=\left|\Uparrow_{A}, \Uparrow_{B}\right\rangle$ and $\left|\Phi_{1}\right\rangle=\left|\Downarrow_{A}, \Downarrow_{B}\right\rangle$, corresponds to an entangled state of the two $\mathrm{Cr}_{7} \mathrm{Ni}$ molecules. Here, electron-nuclear correlations are shown to arise mainly from the opposite chemical shifts of the $\mathrm{H}$ nuclei induced by $\left|\Phi_{1}\right\rangle$ and $\left|\Phi_{2}\right\rangle$. In the second case, the components are instead $\left|\Phi_{1,2}\right\rangle=\left(\left|\Uparrow_{A}, \Downarrow_{B}\right\rangle \pm\left|\Downarrow_{A}, \Uparrow_{B}\right\rangle\right) / \sqrt{2}$. Both the singlet and triplet are characterized by a vanishing expectation value of the electron spin projection, and therefore induce no chemical shift of the nuclei. We find that, as a consequence, the electron-nuclear correlations result from processes that are second-order in the hyper- 


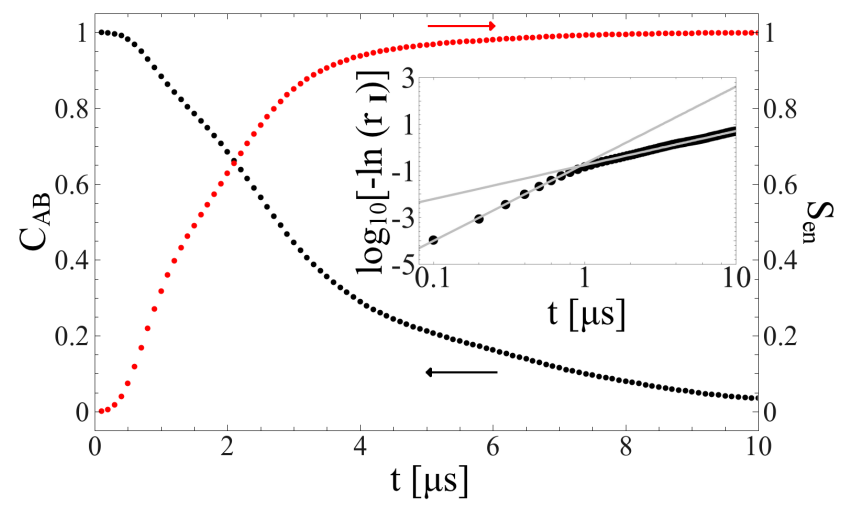

FIG. 2: (color online) Simulated time evolution of the entanglement between $\mathbf{S}_{A}$ and $\mathbf{S}_{B}$, as quantified by the concurrence, $\mathcal{C}_{A B}\left(\rho_{e}^{B S}\right)$ (black circles, left axis). The entanglement between the electron and nuclear spins, for a randomly generated state of the nuclear bath $|\mathbf{I}\rangle$, is quantified by the von Neumann entropy (red circles, right axis). Figure inset: calculated time-dependence of $r_{\mathbf{I}}$ (black circles) and fitting curves $f(t)=\exp \left\{-\left(t / \tau_{d}\right)^{\alpha}\right\}$ (gray lines), with $\alpha=3.31$ and $\tau_{d}=0.70 \mu \mathrm{s}$ and $\alpha=1.46$ and $\tau_{d}=0.75 \mu \mathrm{s}$.

fine interaction (i.e. electron-spin mediated transitions between pairs of nuclei) and selectively involve the $\mathrm{F} \mathrm{nu}$ clei, localized close to the electron spins.

The model - In the $\left(\mathrm{Cr}_{7} \mathrm{Ni}\right)_{2}$ dimer, the spin system and environment consist of $N_{e}=16$ electron spins and $N_{n}=312$ nuclear spins, respectively (Fig. 11(b)). Each $\mathrm{Cr}_{7} \mathrm{Ni}$ wheel is modeled by a ring of eight electron spins one for each of the magnetic ions - with $s_{1-7}=s_{\mathrm{Cr}}=3 / 2$ and $s_{8}=s_{\mathrm{Ni}}=1$. The two lowest eigenstates of each single-ring Hamiltonian form a Kramers doublet: $\left|\Uparrow_{\chi}\right\rangle \equiv$ $\left|S_{\chi}=1 / 2, M_{\chi}=1 / 2\right\rangle$ and $\left|\Downarrow_{\chi}\right\rangle \equiv\left|S_{\chi}=1 / 2, M_{\chi}=-1 / 2\right\rangle$, where $\chi=A, B$. At zero magnetic field this doublet is separated from the first excited quadruplet by an energy gap of $\Delta \simeq 20 \mathrm{~K}[1$. At temperatures $T \ll \Delta$, the state and the couplings of each ring can thus be mapped onto those of an effective $1 / 2$ spin. This also applies to the intermolecular exchange induced by the bipyridine linker in the $\left(\mathrm{Cr}_{7} \mathrm{Ni}\right)_{2}$ dimer (Fig. 11(b)) 1], and to the hyperfine couplings to the nuclear spins [21]. In the presence of an applied magnetic field the effective two-spin Hamiltonian reads:

$$
\mathcal{H}_{A B}=J_{A B} \mathbf{S}_{A} \cdot \mathbf{S}_{B}+g \mu_{B} \sum_{\chi=A, B} \mathbf{S}_{\chi} \cdot \mathbf{B}
$$

with $J_{A B} \ll \Delta$. The spin bath consists of $N_{n}=312$ nuclear spins $\left(6 \mathrm{~F}\right.$ and $306 \mathrm{H}$, with $\left.I_{\mathrm{F}}=I_{\mathrm{H}}=1 / 2\right)$, whose positions are derived from X-ray crystallography. The nuclear spins $\mathbf{I}_{k}$ interact with each other and with the electron spins via dipole-dipole interactions, which are accounted for within the point-dipole approximation.

Energy relaxation of the electron spin state, arising from transitions between different eigenstates $|S, M\rangle$ (with $\mathbf{S}=\mathbf{S}_{A}+\mathbf{S}_{B}$ ), is strongly inhibited, being the dif- ferences between the eigenvalues of $\mathcal{H}_{A B}$ are much larger than both the excitation energies of the nuclear spins and of the hyperfine couplings. In such pure-dephasing regime, the electron-spin decoherence is induced by the dependence of the nuclear-spin dynamics on the electron spin state, and by the resulting electron-nuclear correlations. In particular, an initial state

$$
\left|\Psi_{0}\right\rangle=\left|\Psi_{e 0}\right\rangle \otimes\left|\Psi_{n 0}\right\rangle=\frac{1}{\sqrt{2}}\left(\left|S_{1}, M_{1}\right\rangle+\left|S_{2}, M_{2}\right\rangle\right) \otimes|\mathbf{I}\rangle
$$

(where $\left|\Psi_{e 0}\right\rangle$ and $\left|\Psi_{n 0}\right\rangle=|\mathbf{I}\rangle=\otimes_{k=1}^{N_{n}}\left|I_{k}^{z}\right\rangle$ are the electron and nuclear components, respectively) evolves into

$$
\left|\Psi_{t}\right\rangle=\frac{1}{\sqrt{2}}\left[\left|S_{1}, M_{1}\right\rangle \otimes\left|\mathbf{I}_{1}(t)\right\rangle+e^{i \phi_{\mathbf{I}}(t)}\left|S_{2}, M_{2}\right\rangle \otimes\left|\mathbf{I}_{2}(t)\right\rangle\right] .
$$

Here, $\left|\mathbf{I}_{1}(t)\right\rangle$ and $\left|\mathbf{I}_{2}(t)\right\rangle$ are the states of the nuclear bath conditioned upon the electron spins being in the $\left|S_{1}, M_{1}\right\rangle$ or $\left|S_{2}, M_{2}\right\rangle$ states, respectively. The reduced density matrix of the electron spins is thus given by:

$$
\begin{aligned}
\rho_{e}(t) & =\operatorname{Tr}_{n}\left\{\left|\Psi_{t}\right\rangle\left\langle\Psi_{t}\right|\right\}=\frac{1}{2} \sum_{k=1,2}\left|S_{k}, M_{k}\right\rangle\left\langle S_{k}, M_{k}\right| \\
& +\frac{r_{\mathbf{I}}(t)}{2}\left[\left|S_{2}, M_{2}\right\rangle\left\langle S_{1}, M_{1}\right| e^{i \phi_{\mathbf{I}}(t)}+\text { h.c. }\right],
\end{aligned}
$$

where $r_{\mathbf{I}}=\left|\left\langle\mathbf{I}_{1} \mid \mathbf{I}_{2}\right\rangle\right|$ and $\phi_{\mathbf{I}}=\arg \left\{\left\langle\mathbf{I}_{1} \mid \mathbf{I}_{2}\right\rangle\right\}$ result from the dynamics of the nuclear bath.

This is derived from the effective Hamiltonian 21, 22]:

$$
\mathcal{H}_{\mathrm{eff}}=\sigma_{z}^{e} \otimes \mathcal{H}_{n}^{(e)}+\mathcal{I}^{e} \otimes \mathcal{H}_{n}^{(i)},
$$

being $\left|\Psi_{t}\right\rangle=\exp \left\{-i \mathcal{H}_{\text {eff }} t / \hbar\right\}\left|\Psi_{0}\right\rangle$. Here, the electron and nuclear-spin operators, are given by: $\sigma_{z}^{e} \equiv\left|S_{1}, M_{1}\right\rangle\left\langle S_{1}, M_{1}|-| S_{2}, M_{2}\right\rangle\left\langle S_{2}, M_{2}\right|, \quad \mathcal{I}^{e} \equiv$ $\left|S_{1}, M_{1}\right\rangle\left\langle S_{1}, M_{1}|+| S_{2}, M_{2}\right\rangle\left\langle S_{2}, M_{2}\right|$, and

$$
\mathcal{H}_{n}^{(\alpha)}=\sum_{k=1}^{N_{n}} A_{k}^{(\alpha)} I_{k}^{z}+\sum_{k, l=1}^{N_{n}}\left[B_{k l}^{(\alpha)} I_{k}^{z} I_{l}^{z}+C_{k l}^{(\alpha)} I_{k}^{+} I_{l}^{-}\right],
$$

with $\alpha=e, i$. The intrinsic nuclear Hamiltonian $\mathcal{H}_{n}^{(i)}$ includes the nuclear Zeeman and the secular (i.e. Zeemanenergy conserving) terms of the dipole-dipole interactions between nuclei. The extrinsic Hamiltonian $\mathcal{H}_{n}^{(e)}$ accounts for the Overhauser effect (couplings $A_{k}^{(e)}$ ) and for the interactions between nuclei mediated by virtual excitations of the electron-spins $\left(B_{k}^{(e)}\right.$ and $\left.C_{k}^{(e)}\right)$.

Bell-state decoherence - In order to investigate the decoherence of intermolecular entanglement, we consider the case where the components of $\left|\Psi_{e 0}\right\rangle$ (Eq. 2) are $S_{1}=M_{1}=1$ and $S_{2}=-M_{2}=1$, which corresponds to the spins $\mathbf{S}_{A}$ and $\mathbf{S}_{B}$ being in the Bell state $\left|\Psi_{e 0}\right\rangle=\frac{1}{\sqrt{2}}\left(\left|\Uparrow_{A}, \Uparrow_{B}\right\rangle+\left|\Downarrow_{A}, \Downarrow_{B}\right\rangle\right)$. The simulated build up of correlation between the states of electron and nuclear spins and the resulting loss of entanglement between 


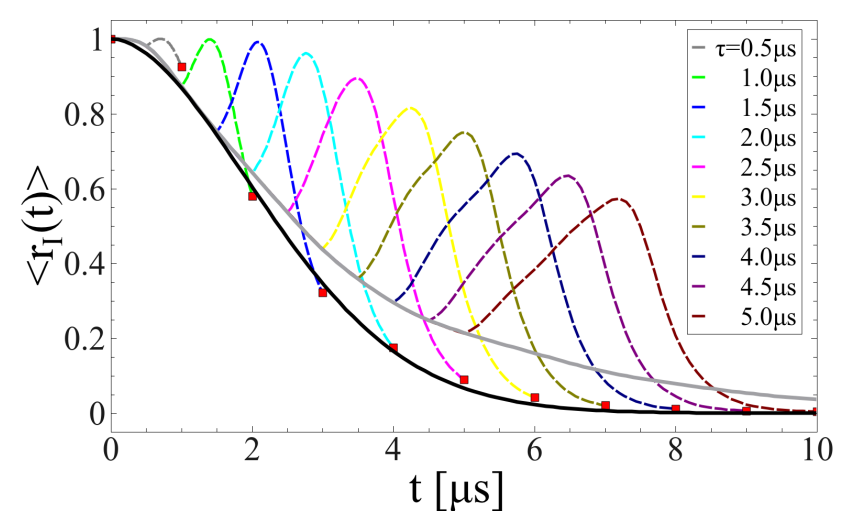

FIG. 3: (color online) Time evolution of $r_{\mathbf{I}}$, averaged over $N=100$ randomly generated initial states $|\mathbf{I}\rangle$, in the absence or in the presence of a $\pi$-pulse applied to the electron spins at $t=\tau$ (solid-gray and dotted curves, respectively; different colors correspond to different values of $\tau$ ). The values $\left\langle r_{\mathbf{I}}(t=2 \tau)\right\rangle$ for different $\tau$ (red squares) are fitted by $f(t)=\exp \left\{-\left(t / \tau_{d}\right)^{\alpha}\right\}$, with $\alpha=1.8$ and $\tau_{d}=2.9 \mu \mathrm{s}$.

$\mathbf{S}_{A}$ and $\mathbf{S}_{B}$ is reported in Fig. 2 The entanglement between two qubits is quantified by the concurrence 23, which, for the density matrix $\rho_{e}$ (Eq. 4), reduces to:

$$
\mathcal{C}_{A B}\left(\rho_{e}\right)=r_{\mathbf{I}}
$$

The von Neumann entropy 23] can be used to quantify entanglement between electron and nuclear spins. Its expression, for $\left|\Psi_{t}\right\rangle$ (Eq. 3), turns out to be:

$$
S_{e n}\left(\rho_{e}\right)=-\frac{1}{2} \sum_{\mu= \pm 1}\left(1+\mu r_{\mathbf{I}}\right) \log _{2}\left[\frac{1}{2}\left(1+\mu r_{\mathbf{I}}\right)\right] .
$$

As $\rho_{e}$ evolves from the initial linear superposition to a statistical mixture of $\left|\Uparrow_{A}, \Uparrow_{B}\right\rangle$ and $\left|\Downarrow_{A}, \Downarrow_{B}\right\rangle, \mathcal{C}_{A B}$ (black circles) and $S_{e n}$ (red circles) tend to 0 and 1, respectively. This clearly shows the mutually exclusive nature of electron-nuclear and intermolecular entanglement. Besides, two distinct regimes can be clearly identified in the entanglement decoherence: for times $t \lesssim 1 \mu \mathrm{s}, r_{\mathbf{I}}(t)$ follows an exponential decay $f(t)=\exp \left\{-\left(t / \tau_{d}\right)^{\alpha}\right\}$, with $\alpha=3.31$ and $\tau_{d}=0.70 \mu \mathrm{s}$; for $t \gtrsim 1 \mu \mathrm{s}$, instead, $\alpha=1.46$ and $\tau_{d}=0.75 \mu$ s (figure inset).

For temperatures much larger than the nuclear Zeeman splitting, the density matrix of the nuclear bath resembles the maximally disordered mixture of states $|\mathbf{I}\rangle: \quad \rho_{n 0}=\sum_{\mathbf{I}}|\mathbf{I}\rangle\langle\mathbf{I}| / 2^{N_{n}}$. While $r_{\mathbf{I}}(t)$ is largely independent on the initial state $|\mathbf{I}\rangle$ of the nuclear bath, this is not the case for the phase $\phi_{\mathbf{I}}$, which depends on the Overhauser field induced by the nuclei (see Eq. 6): $B_{\mathbf{I}}^{n}=-\sum_{k=1}^{N_{n}} A_{k}^{(e)} I_{k}^{z} /\left(\mu_{B} \bar{g}_{z}\right)\left(\bar{g}_{z}\right.$ is the effective $g$ factor of each $\mathrm{Cr}_{7} \mathrm{Ni}$ ring). The static component of the Overhauser field and the resulting inhomogeneous broadening produce a fast dephasing, that can however be eliminated by spin-echo techniques [5. The slow dynamics of the nuclear bath however also results in a fluctuating component of the field, with a potentially irreversible contribution to the phase $\phi_{\mathbf{I}}(t)$ and to the electron-spin decoherence. The simulated dynamics however shows that such contribution affects only marginally entanglement decoherence in the $\left(\mathrm{Cr}_{7} \mathrm{Ni}\right)_{2}$ dimer:

$$
\mathcal{C}_{A B}\left(\rho_{e}^{B S}\right)=\left\langle r_{\mathbf{I}} e^{i \phi_{\mathbf{I}}}\right\rangle \simeq\left\langle r_{\mathbf{I}}\right\rangle
$$

where $0 \leq\left|\left\langle r_{\mathbf{I}} e^{i \phi_{\mathbf{I}}}\right\rangle\right|-\left\langle r_{\mathbf{I}}\right\rangle \leq 0.012$ for $t \leq 10 \mu \mathrm{s}$, and the average is performed on a set of randomly generated initial states $|\mathbf{I}\rangle$.

In order to gain further insight into the decoherence process and underlying dynamics, we isolate the role played by the specific chemical elements and the different physical mechanisms. To this aim, we consider the difference between $\left|\left\langle r_{\mathbf{I}} e^{i \phi_{\mathbf{I}}}\right\rangle\right|$ resulting from the full dynamics and the same quantity obtained by neglecting part of the terms in $\mathcal{H}_{\text {eff }}$. As detailed in Table I the contribution of the $\mathrm{H}(\mathrm{F})$ nuclei to the decoherence of intermolecular entanglement is predominant (negligible). Amongst the hyperfine coupling mechanisms, the effect of the chemical shift (i.e. the renormalizations of the nuclear Zeeman energies induced by the electron spins) largely prevails over the electron-spin mediated interactions between the nuclei. The relative importance of these contributions changes drastically in the case of the singlet-triplet decoherence (see below).

The correlations between electron and nuclear spins described above can be partially reversed by dynamical decoupling schemes [18. In Fig. 3 we show for example the effect on entanglement decoherence of a flip of the electron spins $\mathbf{S}_{\chi=A, B}$ at a time $t=\tau$, that can be induced by an EPR $\pi$-pulse. This ideally transforms the two components of $\left|\Psi_{e 0}\right\rangle$ into one another $\left(\left|\Uparrow_{A}, \Uparrow_{B}\right\rangle \longleftrightarrow\left|\Downarrow_{A}, \Downarrow_{B}\right\rangle\right)$, and thus $\sigma_{z}^{e} \longrightarrow-\sigma_{z}^{e}$ in $\mathcal{H}_{\text {eff }}$ at $t=\tau$. Such inversion of the extrinsic part of the Hamiltonian tends to undo the electron-nuclear correlations; $\left\langle r_{\mathbf{I}}\right\rangle$ correspondingly increase for $t>\tau$, with a maximum localized around $t \simeq \sqrt{2} \tau$ (colored lines) [24]. The above mentioned $\pi$ pulse is used within the Hahn-echo sequence, in order to cancel the effect of inhomogeneous broadening in freeinduction decay experiments and to induce an observable spin echo at time $t=2 \tau$. At that time, electron-nuclear correlations have built up again (red squares), determining values of $\left\langle r_{\mathbf{I}}\right\rangle$ which are lower than those obtained in the absence of the $\pi$-pulse (brown curve). More complex dynamical decoupling schemes are thus required in order to achieve simultaneously the removal of inhomogeneous broadening and electron-nuclear entanglement and to make the latter effect experimentally observable [25].

Singlet-triplet decoherence - In order to complement the results on the entangled state decoherence, we consider the case of a fundamentally different linear combination, namely that between the singlet and triplet states $\left(S_{1}=0, S_{2}=1\right.$ and $M_{1}=M_{2}=0$ in Eq. 2). The reduced density matrix, derived from Eq. 3 , now reads: $\rho_{e}(t)=\frac{1}{2}\left[(1+r)\left|\Uparrow_{A}, \Downarrow_{B}\right\rangle\left\langle\Uparrow_{A}, \Downarrow_{B}|+(1-r)| \Downarrow_{A}, \Uparrow_{B}\right\rangle\left\langle\Downarrow_{A}\right.\right.$ , $\left.\Uparrow_{B} \mid\right]$ (with $r \equiv\left\langle r_{\mathbf{I}} e^{i \phi_{\mathbf{I}}}\right\rangle$ ), and is always factorizable into 


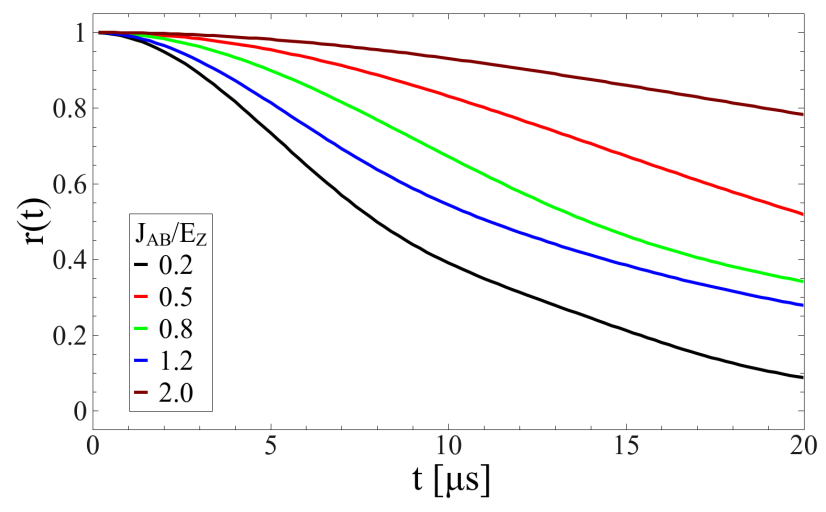

FIG. 4: (color online) Singlet-triplet decoherence as a function of the intermolecular exchange $J_{A B}$ the Zeeman energy $E_{Z}=$ $g \mu_{B} B_{z}$ (Eq. 1). The function $r=\left\langle r_{\mathbf{I}} e^{i \phi_{\mathbf{I}}}\right\rangle$ has been averaged on $N=128$ randomly generated initial states $|\mathbf{I}\rangle$.

\begin{tabular}{|c|cccc|}
\hline & $\begin{array}{c}\text { Chemical } \\
\text { elements }\end{array}$ & $\begin{array}{c}\text { Terms } \\
\text { in } \mathcal{H}_{n}^{(i)}\end{array}$ & $\begin{array}{c}\text { Terms } \\
\text { in } \mathcal{H}_{n}^{(e)}\end{array}$ & $\delta r$ \\
\hline BS & $\mathrm{H}$ & $A_{k}^{(i)}, B_{k l}^{(i)}, C_{k l}^{(i)}$ & $A_{k}^{(e)}$ & 0.02 \\
ST & $\mathrm{F}$ & $A_{k}^{(i)}$ & $A_{k}^{(e)}, B_{k l}^{(e)}, C_{k l}^{(e)}$ & 0.04 \\
\hline
\end{tabular}

TABLE I: Main contributions to the decoherence of the Bell state (BS) and of the singlet-triplet superposition (ST). The key quantity is $\delta r \equiv \max _{t}\left|\left\langle r_{\mathbf{I}}^{*}\right\rangle-\left\langle r_{\mathbf{I}}\right\rangle\right|$, where $r_{\mathbf{I}}^{*}$ is computed by keeping in $\mathcal{H}_{\text {eff }}$ (Eq. 5) only the chemical elements and the terms specified in the central columns; the considered time interval is $0 \leq t \leq 10 \mu \mathrm{s}$.

the states of $\mathbf{S}_{A}$ and $\mathbf{S}_{B}: \mathcal{C}_{A B}\left(\rho_{e}\right)=0$.

The features of the singlet-triplet decoherence are fundamentally different from those emerged in the case of the Bell state. As shown in Fig. 4, the time-evolution of $r$ is strongly dependent on the intermolecular exchange $J_{A B}$ (Eq. 1). In particular, the decoherence rate is on average much smaller than that of the Bell state, and decreases for increasing energy difference between the singlet state and each of the triplet states. An analogous dependence (not shown here) is found with respect to the magnetic field. These features are fully consistent with the identification of transitions between $\mathrm{F}$ nuclei, mediated by virtual transitions of the electron-spin state, as the dominant contribution to the overall decoherence (see Table (I). The dynamics of the $\mathrm{H}$ nuclei, though present, is largely independent on the electron-spin state, and therefore doesn't result in the built up of the electron nuclear correlations. As anticipated in the introduction, such correlations arise in the case of the entangled state from the fact that the two components $\left(\left|\Uparrow_{A}, \Uparrow_{B}\right\rangle\right.$ and $\left.\left|\Downarrow_{A}, \Downarrow_{B}\right\rangle\right)$ give rise to opposite chemical shifts. Instead, the chemical shift induced by the singlet and $M=0$ triplet states vanishes, being $\left\langle S, 0\left|s_{i, z}^{\chi}\right| S, 0\right\rangle=0$. The dependence of the nuclear spin dynamics on the electron state therefore relies essentially on second-order processes and on the contribution of the $\mathrm{F}$ nuclei, that are localized closer to the electron spins of the dimer (Fig. 1(a)).

Conclusions - While a number of additional aspects need to be considered in view of their possible relevance in experiments (for example, those related to the hyperfine interaction with the nuclei in the solvent, or to the intialization and manipulation of the electron spin state of the dimer), some fundamental features have emerged from the presented simulations. In particular, we have identified the clear occurrence of two regimes in the build up of electron-nuclear correlations, and of the resulting decoherence of intermolecular entangled. The comparison with the case of singlet-triplet decoherence shows that the decoherence in the low-energy subspace of the dimer can be induced by distinct chemical elements and physical processes, depending on the components of the initial linear combination. Finally, as demonstrated in other spin systems, dynamical decoupling might allow to disentangle electron and nuclear spins, thus leading to a substantial recovery of the intermolecular coherence.

We are grateful to M. Affronte and V. Bellini for fruitful discussions; we thank M. Rontani and D. Prezzi for a careful reading of the manuscript. We acknowledge financial support from the Italian CNR-INFM under SEED 2008 and the EU under MolSpinQIP.
[1] A. Candini et al., Phys. Rev. Lett. 104, 037203 (2010).

[2] D. Gatteschi et al., Molecular nanomagnets (Oxford University Press, 2007).

[3] W. Wernsdorfer et al., Nature 416, 406 (2002).

[4] G. A. Timco et al., Nature Nanotech. 4, 173 (2009).

[5] A. Ardavan et al., Phys. Rev. Lett. 98, 057201 (2007).

[6] S. Takahashi et al., Phys. Rev. Lett. 102, 087603 (2009).

[7] S. Bertaina et al., Nature 453, 203 (2008).

[8] S. Bertaina et al., Phys. Rev. Lett. 102, 050501 (2009).

[9] C. Schlegel et al., Phys. Rev. Lett. 101, 147203 (2008).

[10] M. N. Leuenberger et al., Nature 410, 789 (2001).

[11] F. Meier et al., Phys. Rev. Lett. 90, 047901 (2003).

[12] F. Troiani et al., Phys. Rev. Lett. 94, 190501 (2005).

[13] M. Trif et al., Phys. Rev. Lett. 101, 217201 (2008).
[14] A. Morello et al., Phys. Rev. Lett. 97, 207206 (2006).

[15] M. N. Leuenberger et al., Phys. Rev. B 61, 1286 (2000).

[16] N. V. Prokof'ev et al., Rep. Prog. Phys. 63, 669 (2000).

[17] W. A. Coish et al., Phys. Status Solidi B 246, 2203 (2009).

[18] L. Viola et al., Phys. Rev. A 58, 2733 (1998).

[19] F. Troiani et al., Nanotechnology 21, 274009 (2010).

[20] W. H. Zurek, Rev. Mod. Phys. 75, 715 (2003).

[21] F. Troiani et al., Phys. Rev. B 77, 054428 (2008).

[22] W. Yao et al., Phys. Rev. B 74, 195301 (2006).

[23] L. Amico et al., Rev. Mod. Phys. 80, 517 (2008).

[24] W. Yao et al., Phys. Rev. Lett. 98, 077602 (2007).

[25] M. J. Biercuk et al., Nature 458, 996 (2009). 